\title{
The effectiveness and value of novel treatments for cystic fibrosis
}

\author{
A summary from the Institute for Clinical and Economic Review's \\ California Technology Assessment Forum
}

Jeffrey A Tice, MD; Karen M Kuntz, ScD; Kael Wherry, PhD; Matt Seidner, BS; David M Rind, MD, MSc; and Steven D Pearson, MD, MSc

Cystic fibrosis (CF) is an autosomal recessive condition caused by mutations in the cystic fibrosis transmembrane conductance regulator (CFTR) gene. The CFTR protein is an ion channel at the cell surface that primarily transports chloride ions across the cell membrane. According to the Cystic Fibrosis Foundation Annual Report, the overall prevalence of $\mathrm{CF}$ in the United States in 2016 was 30,775. ${ }^{1}$

The effect of the disease on patients is profound. The thick secretions cause chronic lung infections, reduced lung function, poor weight gain (due to gastrointestinal dysfunction), diabetes (due to pancreatic damage), and fertility problems. ${ }^{2}$ Patients suffer frequent acute pulmonary exacerbations, leading to repeated hospitalizations and long courses of intravenous (IV) antibiotics that require invasive procedures such as the placement of ports for IV access and repeated absence from school and work. Patients and their families frequently spend several hours every day on treatments intended to help clear the lungs of secretions. The disease is progressive over time, and patients may require lung transplantation to live. Although $\mathrm{CF}$ is uncommon, it represents a substantial economic burden. In 2013, CF-related hospital costs alone were estimated to exceed $\$ 1.1$ billion. $^{3}$
Over the past decade, the U.S. Food and Drug Administration (FDA) has approved several novel drugs that directly modulate the abnormal CFTR protein. The drugs target specific classes of CFTR gene mutations. The FDA approved the oral triple therapy, Trikafta (elexacaftor/tezacaftor/ ivacaftor, Vertex Pharmaceuticals) on October 21, 2019, for the treatment of $\mathrm{CF}$ in patients aged 12 years and older who have at least 1 copy of the F508del mutation. This mutation is present in approximately $90 \%$ of patients with $\mathrm{CF}$. The 3 other FDA-approved modulator therapies are Kalydeco (ivacaftor), Orkambi (lumacaftor/ivacaftor), and Symdeko (tezacaftor/ivacaftor).

The Institute for Clinical and Economic Review (ICER) conducted a systematic literature review and cost-effectiveness analysis to evaluate the health and economic outcomes of these CFTR modulator therapies. Complete details of ICER's systematic literature search and protocol, as well as the methodology and model structure for the economic evaluation, are available on ICER's website. Here, we present the summary of our findings and highlights of the policy discussion with key stakeholders held at a public meeting of the California Technology Assessment Forum on August 27, 2020. The detailed report is available

\author{
Author affiliations \\ Jeffrey A Tice, MD, Division of General \\ Internal Medicine, University of California, \\ San Francisco. Karen M Kuntz, ScD, and \\ Kael Wherry, PhD, School of Public Health, \\ University of Minnesota. Matt Seidner, BS; \\ David M Rind, MD, MSc; and Steven D \\ Pearson, MD, MSc, Institute for Clinical \\ and Economic Review, Boston, MA. \\ AUTHOR CORRESPONDENCE: \\ Jeffrey A Tice, jeff.tice@ucsf.edu \\ J Manag Care Spec Pharm. \\ 2021;27(2):276-80 \\ Copyright $\odot 2021$, Academy of Managed \\ Care Pharmacy. All rights reserved.
}

on the ICER website at http://icerorg.wpengine.com/wp-content/ uploads/2020/08/ICER_CF_Final_ Report_092320.pdf.

\section{Summary of Findings}

\section{CLINICAL EFFECTIVENESS}

The primary outcome in the trials was the absolute change in the percent predicted forced expiratory volume in 1 second (ppFEV1), and quality of life was assessed using the respiratory domain of the Cystic Fibrosis 


\section{FIGURE 1 Network Meta-Analysis Results for ppFEV1 Comparing CF Modulator Therapy to Placebo}

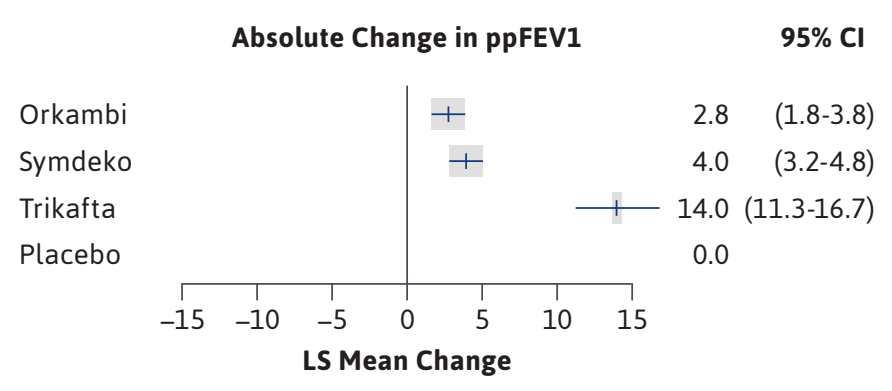

$C F=$ cystic fibrosis; $L S=$ least squares; $p p F E V 1=$ percent predicted forced expiratory volume in 1 second.

Questionnaire Revised (CFQ-R), which has a minimum clinically important difference of 4 points.

For patients homozygous for the F508del mutation, there was 1 head-to-head trial between Trikafta and Symdeko. ${ }^{4}$ Compared with Symdeko, the ppFEV1 in patients randomized to Trikafta was 10.0 points higher at 4 weeks $(95 \%$ $\mathrm{CI}=7.4-12.6, \mathrm{P}<0.001)$. Quality of life as assessed by the respiratory domain of the CFQ-R was 17.4 points higher in the Trikafta group (95\% CI=11.8-23, $\mathrm{P}<0.001)$. A network meta-analysis including 3 additional trials ${ }^{5-7}$ in this population demonstrated significantly greater improvements for Trikafta in ppFEV1 (Figure 1), quality of life, and sweat chloride levels compared with Symdeko, Orkambi, and placebo.

There are no published studies evaluating the safety and efficacy of Trikafta in patients heterozygous for the F508del mutation and a residual function mutation. However, Trikafta is comprised of Symdeko plus another modulator (elexacaftor), and it primarily targets the abnormal protein formed by the F508del mutation. Unless elexacaftor interferes with the mechanism of action of ivacaftor and/or tezacaftor or introduces new, significant adverse events, Trikafta should be at least as effective as Symdeko in this population. In randomized trials versus placebo, Symdeko increased ppFEV1 by $6.8 \%$, reduced acute pulmonary exacerbations by $46 \%$, and improved the respiratory domain of the CFQ-R by 11.1 points. $^{8}$

There is 1 randomized trial of Trikafta compared with placebo in patients heterozygous for the F508del mutation and a minimal function mutation. ${ }^{9}$ The ppFEV1 of patients on Trikafta was 14.3 points higher at 24 weeks $(P<0.001)$. Quality of life as assessed by the respiratory domain of the CFQ-R was 20.2 points higher in the Trikafta group $(\mathrm{P}<0.001)$.

Serious adverse events (AEs), as defined by the studies, commonly occurred at the same or lower rates among those taking Trikafta or other CFTRs than those taking placebo, including AEs ascribed to the drugs. No deaths during CFTR modulator trials were related to the drugs.

\section{LIMITATIONS OF THE CLINICAL EVIDENCE}

$\mathrm{CF}$ is a chronic disease that affects patients every day of their lives. The 2 pivotal clinical trials of Trikafta lasted 4 and 24 weeks, respectively, which is not long enough to provide stable estimates for the long-term effect of Trikafta. In addition, there are likely differences in the long-term benefits of Trikafta based on patients' ages at initiation of therapy and the severity of their CF symptoms at that time. Finally, in patients heterozygous for the F508del mutation and a residual function mutation, there are no data on Trikafta, although we do have data on Symdeko, which includes 2 of the 3 drugs in Trikafta.

\section{LONG-TERM COST-EFFECTIVENESS}

We estimated the lifetime effectiveness and cost-effectiveness of CFTR modulator treatments plus best supportive care for $\mathrm{CF}$ patients. We modeled 4 different populations based on mutation status. For patients who are candidates for Kalydeco only based on current indications, we compared Kalydeco plus best supportive care with best supportive care alone. For patients who are homozygous for the F508del mutation and patients who are heterozygous for the F508del mutation with a residual function mutation, we compared Trikafta plus best supportive care, Symdeko plus best supportive care, Orkambi plus best supportive care (for base-case results and threshold price only), and best supportive care alone. For patients who are heterozygous for the F508del mutation with a minimal function mutation, we compared Trikafta plus best supportive care with best supportive care alone.

The model was informed by the ICER network metaanalysis of key clinical trials, previous relevant economic models, systematic literature reviews, and input from stakeholders. The outcomes of interest included the incremental cost per quality-adjusted life-year (QALY) gained, life-years gained, equal value of life-years gained (evLYG), and cost per pulmonary exacerbation prevented. Full details on ICER's cost-effectiveness analysis and model are available on ICER's website at http://icerorg.wpengine. com/wp-content/uploads/2020/08/ICER CF Final Report 092320.pdf.

Table 1 summarizes the cost-effectiveness results for each of the drugs compared with best supportive care in 


\section{TABLE 1}

Incremental Cost-Effectiveness Ratios Compared with BSC for the Base Case

\begin{tabular}{|c|c|c|c|c|}
\hline Treatment vs. BSC & $\begin{array}{c}\text { Cost Per } \\
\text { QALY Gained, \$ }\end{array}$ & $\begin{array}{l}\text { Cost Per } \\
\text { evLYG, } \$\end{array}$ & $\begin{array}{c}\text { Cost Per } \\
\text { LY Gained, \$ }\end{array}$ & $\begin{array}{c}\text { Cost Per } \\
\text { PEx Averted, \$ }\end{array}$ \\
\hline \multicolumn{5}{|c|}{ Population 1: Eligible for Kalydeco monotherapy only } \\
\hline Kalydeco plus BSC & $1,370,000$ & $1,180,000$ & $1,450,000$ & 737,000 \\
\hline \multicolumn{5}{|c|}{ Population 2: Homozygous for the F508del mutation } \\
\hline Orkambi plus BSC & $1,480,000$ & $1,360,000$ & $1,440,000$ & 614,000 \\
\hline Symdeko plus BSC & $1,380,000$ & $1,200,000$ & $1,400,000$ & 621,000 \\
\hline Trikafta plus BSC & $1,160,000$ & $1,040,000$ & $1,230,000$ & 675,000 \\
\hline \multicolumn{5}{|c|}{ Population 3: Heterozygous for F508del with residual function mutation } \\
\hline Symdeko plus BSC & $1,340,000$ & $1,100,000$ & $1,390,000$ & 549,000 \\
\hline Trikafta plus BSC & $1,100,000$ & 951,000 & $1,210,000$ & 539,000 \\
\hline \multicolumn{5}{|c|}{ Population 4: Heterozygous for F508del with minimal function mutation } \\
\hline Trikafta plus BSC & $1,050,000$ & 877,000 & 990,000 & 565,000 \\
\hline
\end{tabular}

each of the populations for which the drug has an FDA indication. In all cases, the cost per QALY gained is greater than \$1 million, which is substantially higher than cost-effectiveness thresholds in the United States and the rest of the world. To demonstrate how extreme the pricing is, we performed a hypothetical cost-effectiveness analysis assuming that Trikafta cures CF (normal life expectancy, normal quality of life, no costs due to CF other than Trikafta). At its current price, the incremental cost-effectiveness ratio would be $\$ 612,000$ per QALY, which remains far above commonly accepted costeffectiveness thresholds.

\section{LIMITATIONS OF THE COST-EFFECTIVENESS MODEL}

We used ppFEV1 as the primary marker of lung function to characterize the progression of $\mathrm{CF}$ over time. As with any surrogate marker of disease, ppFEV1 is not a perfect marker for progression. In addition, limited evidence exists about the effect of the CFTR modulators on the ability of individuals to work or attend school, or the degree to which caregiver burden is reduced by CFTR modulator treatment. In addition, we only had short-term measures of drug effect and had to make assumptions about their effect over the lifetime of the patient. Finally, we used trial-based estimates of discontinuation of these therapies to be consistent with the efficacy estimates; real-world patterns of discontinuation may differ from these.

\section{Policy Discussion}

The California Technology Assessment Forum (CTAF, https://icer-review. org/programs/ctaf/) is one of the independent appraisal committees convened by ICER to engage in the public deliberation of the evidence on clinical and cost-effectiveness of health care interventions. CTAF is composed of medical evidence experts, including practicing clinicians, methodologists, and leaders in patient engagement and advocacy.
Their deliberation includes input from clinical experts and patient representatives specific to the condition under review, as well as formal comment from manufacturers and the public. A policy roundtable concludes each meeting during which representatives from insurers and manufacturers join clinical experts and patient representatives to discuss how best to apply the findings of the evidence to clinical practice, insurance coverage, and pricing negotiations.

The ICER report on treatments for CF was the subject of a CTAF meeting on August 27, 2020. Following the discussion, the CTAF panel members deliberated on key questions raised by ICER's report. The results of their votes on the clinical evidence are as follows:

1. The panel voted $14-0$ that the clinical evidence was adequate to demonstrate greater net health benefit for Trikafta compared with both best supportive care and Symdeko for patients homozygous for the F508del mutation.

2. The panel voted 13-1 that there was adequate evidence to demonstrate greater net health benefit for Trikafta compared with best supportive care for patients heterozygous for the F508del mutation and a residual function mutation.

3. The panel voted 6-8 that there was adequate evidence to demonstrate greater net health benefit for Trikafta compared with Symdeko for patients heterozygous for the F508del mutation and a residual function mutation.

4. The panel voted 14-0 that the evidence was adequate evidence to demonstrate greater net health benefit for Trikafta compared with best supportive care for patients heterozygous for the F508del mutation and a minimal function mutation. 


\section{TABLE 2 Votes on "Other Benefits" that Are Not Adequately Captured in the Base-Case Cost-Effectiveness Model ${ }^{a}$}

\begin{tabular}{l|c}
\hline \hline $\begin{array}{l}\text { This intervention will significantly reduce caregiver or } \\
\text { broader family burden. }\end{array}$ & $13 / 13$ \\
\hline $\begin{array}{l}\text { This intervention offers a novel mechanism of action } \\
\text { or approach that will allow successful treatment of } \\
\text { many patients for whom other available treatments } \\
\text { have failed. }\end{array}$ & $12 / 13$ \\
\hline $\begin{array}{l}\text { This intervention will have a significant impact on } \\
\text { improving patients' ability to return to work and/or } \\
\text { their overall productivity. }\end{array}$ & $13 / 13$ \\
\hline $\begin{array}{l}\text { This intervention will have a significant positive } \\
\text { impact outside the family, including on schools and/or } \\
\text { communities. }\end{array}$ & $11 / 13$ \\
$\begin{array}{l}\text { There are other important benefits or disadvantages } \\
\text { that should have an important role in judgments of } \\
\text { the value of this intervention }\end{array}$ & $6 / 13$ \\
$\begin{array}{l}{ }^{a} \text { Only } 13 \text { CTAF panelist votes were tallied due to a malfunction with the voting } \\
\text { technology. } \\
\text { CTAF = California Technology Assessment Forum. }\end{array}$
\end{tabular}

The CEPAC panel also voted on "other potential benefits" and "contextual considerations" as part of a process intended to signal to policymakers whether there are important considerations when making judgments about long-term value for money not adequately captured in analyses of clinical effectiveness and/or cost-effectiveness. The results of these votes are shown in Table 2 and Table 3. They highlight several factors beyond the results of cost-effectiveness modeling that the CEPAC panel felt were particularly important for judgments of overall long-term value for money, including the reduction in caregiver and family burden, the novel mechanism of action of the Trikafta, and the substantial lifetime burden of illness suffered by patients with cystic fibrosis.

As described in ICER's Value Assessment Framework, questions on long-term value for money are subject to a value vote when incremental cost-effectiveness ratios for the interventions of interest are between $\$ 50,000$ and $\$ 175,000$ per QALY in the primary base-case analysis. The base-case estimates of the cost per QALY for Trikafta in all populations exceeded the higher end of this range; therefore, the treatment was deemed "low long-term value for money at current prices" without a vote unless CTAF determined in its discussion that the evidence report basecase analysis did not adequately reflect the most probable incremental cost-effectiveness ratio for Trikafta.

The policy roundtable discussion explored how best to translate the evidence and additional considerations

\section{TABLE 3 Votes on "Contextual Considerations Important in Assessing Long-Term Value for Money"}

\begin{tabular}{l|c}
\hline \hline $\begin{array}{l}\text { This intervention is intended for the care of individuals } \\
\text { with a condition of particularly high severity in terms of } \\
\text { impact on length of life and/or quality of life. }\end{array}$ & $14 / 14$ \\
\hline $\begin{array}{l}\text { This intervention is intended for the care of individuals } \\
\text { with a condition that represents a particularly high } \\
\text { lifetime burden of illness. }\end{array}$ & $14 / 14$ \\
\hline $\begin{array}{l}\text { This intervention is the first to offer any improvement for } \\
\text { patients with this condition. }\end{array}$ & $8 / 14$ \\
$\begin{array}{l}\text { Compared with best supportive care, there is significant } \\
\text { uncertainty about the long-term risk of serious side } \\
\text { effects of this intervention. }\end{array}$ & $11 / 14$ \\
$\begin{array}{l}\text { Compared with best supportive care, there is significant } \\
\text { uncertainty about the magnitude or durability of the }\end{array}$ & $10 / 14$ \\
$\begin{array}{l}\text { long-term benefits of this intervention. } \\
\begin{array}{l}\text { There are additional contextual considerations that } \\
\text { should have an important role in judgments of the value } \\
\text { of this intervention. }\end{array}\end{array}$ & $1 / 14$ \\
\hline
\end{tabular}

into clinical practice and into pricing and insurance coverage policies. The full set of policy recommendations can be found in the Final Evidence Report on the ICER website: http://icerorg.wpengine.com/wp-content/ uploads/2020/08/ICER_CF_Final_Report_092320.pdf. Several key policy recommendations focused on Trikafta are as follows:

- The manufacturer should lower the price of Trikafta to align fairly with its demonstrated benefits.

- Public and private payers should continue to affirm their commitment to provide access to Trikafta and should remove superfluous requirements for coverage approval and continuation.

- For Trikafta, it is important for payers to seek to control costs without using access restrictions as a key feature of negotiation. Patients and their families need to know that insurers will help them receive these new drugs.

- Insurer prior authorization criteria should be based on clinical evidence, specialty society guidelines, and input from clinical experts and patient groups. The process for authorization should be clear and efficient for providers. Considerations for prior authorization include the following:

- Patient eligibility (diagnosis): Proof of CFTR mutation status (at least 1 F508del mutation) should be required for initial coverage but not for requalification, since CFTR modulator treatments do not permanently modify the genome. 
- Patient eligibility (age): Age 12 years or older is the age threshold supported by direct clinical evidence and codified at the time Trikafta was first approved by the FDA. Given that there is no a priori clinical rationale that younger patients would not benefit and that more recent evidence suggests that the FDA may soon expand the label to a younger population, payers should establish clear pathways for exceptions and consider coverage for younger patients meeting other clinical criteria. It is reasonable for payers to require these exception requests be made by a practicing CF expert.

- Provider criteria: Given the complexity of managing $\mathrm{CF}$ and of ensuring appropriate overall care for patients, it is reasonable for insurers to require that Trikafta be prescribed by a $\mathrm{CF}$ clinical expert.

- Step therapy: In general, step therapy should not be required for patients who qualify for Trikafta because Trikafta is clearly superior to Symdeko and Orkambi in most patients who carry the F508del mutation.

- Renewal criteria: Provider attestation of clinical benefit is sufficient given that there is no defined standard for clinical response to treatment.

\section{DISCLOSURES}

Funding for this summary was contributed by Arnold Ventures, California Health Care Foundation, Harvard Pilgrim Health Care, and Kaiser Foundation Health Plan to the Institute for Clinical and Economic Review (ICER), an independent organization that evaluates the evidence on the value of health care interventions. ICER's annual policy summit is supported by dues from Aetna, America's Health Insurance Plans, Anthem, Allergan, Alnylam, AstraZeneca, Biogen, Blue Shield of CA, BoehringerIngelheim, Cambia Health Services, CVS, Editas, Express Scripts, Genentech/ Roche, GlaxoSmithKline, Harvard Pilgrim, Health Care Service Corporation, HealthFirst, Health Partners, Johnson \& Johnson (Janssen), Kaiser Permanente, LEO Pharma, Mallinckrodt, Merck, Novartis, National Pharmaceutical Council, Pfizer, Premera, Prime Therapeutics, Regeneron, Sanofi, Spark Therapeutics, and United Healthcare.

Seidner, Rind, and Pearson are employed by ICER. Tice reports contracts to his institution, University of California, San Francisco, from ICER during the conduct of this study. Wherry has nothing to disclose.

\section{ACKNOWLEDGMENTS}

The authors thank Noemi Fluetsch and Avery McKenna for their contributions to this report.

\section{REFERENCES}

1. Cystic Fibrosis Foundation. Cystic Fibrosis Foundation Patient Registry 2018 Annual Data Report. 2019. Accessed January 9, 2021. https://www.cff.org/ Research/Researcher-Resources/ Patient-Registry/2018-Patient-RegistryAnnual-Data-Report.pdf
2. Patel S, Sinha IP, Dwan K, Echevarria C, Schechter M, Southern KW. Potentiators (specific therapies for class III and IV mutations) for cystic fibrosis. Cochrane Database Syst Rev. 2015(3):CD009841.

3. Agrawal A, Mehta D, Sikachi R, Du D, Wang J. Nationwide trends of hospitalizations for cystic fibrosis in the United States from 2003 to 2013. Intractable Rare Dis Res. 2017;6(3):191-98.

4. Heijerman H, McKone E, Downey D, et al. Efficacy and safety of the elexacaftor plus tezacaftor plus ivacaftor combination regimen in people with cystic fibrosis homozygous for the F508del mutation: a double-blink, randomised, phase 3 trial. Lancet. 2019;394(10212):1940-48.

5. Keating D, Marigowda G, Burr L, et al. VX-445-Tezacaftor-ivacaftor in patients with cystic fibrosis and one or two Phe508del alleles. N Engl J Med. 2018;379(17):1612-20.

6. Taylor-Cousar JL, Jain M, Barto TL, et al. Lumacaftor/ivacaftor in patients with cystic fibrosis and advanced lung disease homozygous for F508del-CFTR. J Cyst Fibros. 2018;17(2):228-35.

7. Wainwright CE, Elborn JS, Ramsey BW, et al. Lumacaftor-ivacaftor in patients with cystic fibrosis homozygous for Phe508del CFTR. N Engl J Med. 2015;373(3):220-31.

8. Rowe SM, Daines C, Ringshausen FC, et al. Tezacaftor-ivacaftor in residualfunction heterozygotes with cystic fibrosis. N Engl J Med. 2017;377(21):2024-35.

9. Middleton PG, Mall M, Drevinek P, et al. Elexacaftor-tezacaftor-ivacaftor for cystic fibrosis with a single Phe508del allele. N Engl J Med. 2019;381(19):1809-19. 